potential risk. It is also our responsibility to educate families of the signs of hypoglycaemia so they can be aware, particularly in the younger children who appear to be at increased risk.

\section{G417(P) DROPLET DIGITAL POLYMERASE CHAIN REACTION (DDPCR) ASSESSMENT OF DEEP MOLECULAR RESPONSE IN CHRONIC MYELOID LEUKAEMIA (CML)}

M Jyothish, S Akiki. School of Cancer Sciences, University of Birmingham, Birmingham, UK

10.1136/archdischild-2015-308599.371

Molecular monitoring of BCR-ABL1 transcript levels is central to the effective clinical management of patients with CML. Standardised Real time quantitative PCR (RT-qPCR) is currently used to measure the depth and durability of molecular response and forms the basis of the European Leukaemia Net (ELN) treatment recommendations. Close monitoring of patients who come off treatment using maximum sensitivity techniques, is vital in order to ensure early therapeutic intervention, should a BCR-ABL1 clone re-emerge. However, RT-qPCR is susceptible to fluctuations at the limits of its sensitivity, thus limiting its accuracy in assessing complete molecular response. Droplet Digital PCR technology is a new digital PCR technique where the test sample into many individual real-time PCR reactions, where some reactions contain the target molecule (positive) while others (negative) do not. The proportion of negative reactions is used to calculate an absolute count of the number of target molecules in the sample, without reference to external standards. This method should therefore enable increased accuracy and absolute quantification of target molecules, in comparison to the RTqPCR technique. The aim of this study was to assess the potential of Droplet digital PCR (ddPCR) to improve the sensitivity and precision of the assessment of deep molecular response in patients with CML. A series of 21 patients previously determined to have achieved major molecular response (MMR) using RT-qPCR, were retrospectively monitored for BCR-ABL1 transcripts using ddPCR. A comparison of BCR-ABL1 to ABL1 ratios obtained by ddPCR and RT-qPCR suggested that in $63 \%$ of patients ddPCR was more sensitive than RT-qPCR, while in $28 \%$ of patients BCR-ABL1 transcript levels were better detected by RT-qPCR. This study demonstrates that ddPCR has the potential to precisely measure changes in minimal residual disease at the limits of detection of current RT-qPCR assays. Assessment of deep molecular response using ddPCR will help to accurately discriminate patients achieving a sustainable response and could therefore be considered for treatment cessation studies from those patients in whom BCR-ABL1 transcripts are likely to re emerge.

\section{G418(P) PAEDIATRIC ONCOLOGY JUNIOR DOCTOR CONFIDENCE SURVEY}

C Parfitt. Gloucestershire Royal Hospital, Gloucester, UK

10.1136/archdischild-2015-308599.372

Aims Following a Paediatric Oncology MDT event reviewing themes of 'Teamwork and Integration' the department was keen to explore how confident trainees are at managing Oncology patients and how teamwork could be improved within the department.
Methods In October 2013 a Confidence Survey was disseminated to the current cohort of SHOs and SpRs. Confidence and support questions were answered on a scale rating system, and space given for additional comments. Results were analysed and change implemented in January 2014 with re-audit in August 2014.

Results Initial survey numbers were small with a poor response rate $(39 \%)$. However in those that responded clear themes emerged.

No trainees were aware of when the Oncology MDT meeting occurs, and none felt part of the Oncology team. 66\% of trainees felt Oncology teaching and clinical exposure failed to meet their curriculum needs.

In general the registrars felt more confident managing patients and reassuringly most felt supported by the team.

In the free-flow comments, there was demand to attend oncology clinics and spend more time on the Oncology Unit. Trainees requested teaching expansion beyond febrile neutropenia and to include practical advice regarding management plans.

Based on this feedback changes were implemented. This included clearer highlighting of the Oncology department, introduction of weekly ward round attendance for trainees including once a week grand round teaching, teaching sessions allocated to cover curriculum topics, introduction of weekend plan stickers and an individual patient summary front sheet.

Re-audit in August 2014 showed an improved response rate $(50 \%)$. There was an improvement in awareness of MDT meetings and feeling of teamwork. $70 \%$ of trainees felt Oncology teaching and exposure met their needs.

Conclusions The changes clearly show an improvement in both confidence and feeling of support by the Oncology Team. By changing the clinical environment through introduction of daily ward rounds the team is better integrated and ad-hoc teaching has improved. The introduction of the summary sheets and weekend plans has helped on a day-to-day practical basis of managing patients, and has also helped junior doctors gain a better understanding of different conditions and specific management plans.

\section{British Paediatric Allergy Immunology and Infection and British Association for Community Child Health}

\section{G419 "WE CAN'T CHANGE ANYTHING THAT'S HAPPENED IN THEIR PAST, BUT WHAT WE CAN DO IS HELP THEM WITH SOME OF THE INTERNAL MESS THEY'VE BEEN LEFT WITH TO DEAL WITH": HEALTHCARE PROFESSIONALS' EXPERIENCES OF THE INITIAL HEALTHCARE ASSESSMENTS FOR UNACCOMPANIED ASYLUM SEEKING CHILDREN AND YOUNG PEOPLE}

C Shortall, H Bedford. Institute of Child Health, University College London, London, UK

\subsection{6/archdischild-2015-308599.373}

Currently 1,860 Unaccompanied Asylum Seeking Children and Young People (UASCYPs) are being "Looked-After" in England; NICE guidance on how best to meet their needs has called for cultural competency and highlighted a deficit in an applicable evidence-base. This study contributes to the evidence-base for the healthcare needs of UACYPs and factors which influence them within the context of the Initial Healthcare Assessment (IHA). 